IS There ChaOS IN THE WORLd ECONOMY?

A NonParametric TeSt USIng Consistent Standard Errors

by

Mototsugu Shintani and Oliver Linton

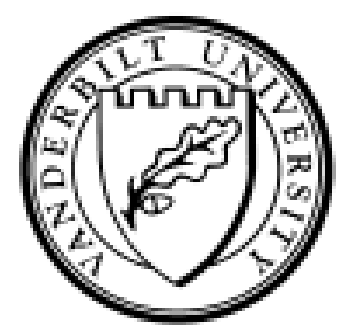

Working Paper No. 01-W11

June 2001

DEPARTMENT OF ECONOMICS

VANDERBILT UNIVERSITY

NASHVILLE, TN 37235

www.vanderbilt.edu/econ 


\title{
Is There Chaos in the World Economy? A Nonparametric Test Using
}

\section{Consistent Standard Errors*}

\author{
Mototsugu Shintani ${ }^{\dagger}$ \\ Oliver Linton $\ddagger$ \\ Vanderbilt University London School of Economics
}

September 2000

Revised: June 2001

\begin{abstract}
A positive Lyapunov exponent is one practical definition of chaos. We develop a formal test for chaos in a noisy system based on the consistent standard errors of the nonparametric Lyapunov exponent estimators. When our procedures are applied to international real output series, the hypothesis of the positive Lyapunov exponent is significantly rejected in many cases. One possible interpretation of this result is that the traditional exogenous models are better able to explain business cycle fluctuations than is the chaotic endogenous approach. However, our results are subject to a number of caveats, in particular our results could have been influenced by small sample bias, high noise level, incorrect filtering, and long memory of the data.
\end{abstract}

Keywords: Artificial neural networks, Business cycles, Local polynomial regression, Nonlinear dynamics, Nonlinear time series. JEL classification: C14, E32.

${ }^{*}$ The authors thank Barb Bailey and Doug Nychka for providing their computer program. We would especially like to thank Buz Brock for his detailed comments and encouragement that has helped us clarify our thinking. We also thank two anonymous referees, Ron Gallant, Dominique Guégan, Peter Phillips, Tadashi Shigoka and Yoon Whang for comments, and the National Science foundation for financial support.

${ }^{\dagger}$ Department of Economics, Vanderbilt University, Box 1819 Station B, Nashville, TN 37235, USA. e-mail: mototsugu.shintani@vanderbilt.edu

${ }^{\ddagger}$ Department of Economics, London School of Economics, Houghton Street, London WC2A 2AE, United Kingdom. e-mail: lintono@lse.ac.uk 


\section{Introduction}

How to interpret business cycle fluctuations has been one of the most important issues in economics. The standard way of incorporating business cycles into macroeconomic models is to treat cycles as a temporary divergence from the steady state (or steady growth path) caused by exogenous shocks such as policy shifts and technological change. The idea of duplicating the actual business cycle fluctuations by using impulse responses of uncorrelated random shocks originates with Slutzky's (1927) work; this approach was popularized by Sims (1980), who put more emphasis on identifying shocks in a multivariate system. Impulse response analysis has now become a basic tool for evaluating the shock propagation mechanism of the dynamic stochastic general equilibrium (DSGE) models [which include the stochastic growth model/real business cycle model], using the linear approximation method based on the assumption of the economy being close to a unique and globally stable steady state. (See Campbell (1994) and Cooley (1995), for example.)

Alternatively, macroeconomists have long realized that a certain class of deterministic nonlinear systems was capable of producing a self-sustained fluctuation without any shocks from outside of the model. The limit cycle model once attracted researchers, including Kaldor (1940) and Hicks (1950), but such an approach was confronted by the simple fact that the actual fluctuation of real data was apparently not exactly periodic. In the early 1980s, the discovery of chaotic dynamics in the field of natural science helped to prompt a revival of endogenous business cycle modeling. A group of researchers, including Benhabib and Nishimura (1979), Day (1982) and Grandmont (1985), developed many examples of deterministic economic models that could generate nonperiodic fluctuations. Indeed, recent studies (Brock and Hommes (1997), (1998)) have shown that routes to chaos can arise in the traditional expectation models such as the cobweb model and the asset pricing model by the introduction of heterogeneous beliefs. These theoretical developments revealed that, depending on the choice of parameter values, most economic models can produce either stable solutions or complex solutions including chaos. For this reason, the theoretical analyses have 
been followed by an active research program to find empirical evidence of chaos as a source of the business cycles. However, it seems fair to say that the currently available evidence on chaos in the macroeconomic time series is rather ambiguous. For example, with the correlation dimension, little evidence consistent with low-dimensional chaos in real output series was found by Brock (1986) and Brock and Sayers (1988) for the U.S., Frank and Stengos (1988) for Canada, and Frank, Gençay and Stengos (1988) for Germany, Italy, Japan and the United Kingdom. On the other hand, based on the correlation dimension, Barnett and Chen (1988) and DeCoster and Mitchell (1991) detected evidence of chaos in U.S. monetary aggregates.

The purpose of this paper is to reinvestigate these alternative views of business cycles by employing the recently developed test for chaos based on nonparametric regression techniques. To be more specific, we focus on the real output series from various countries examined by Brock (1986), Frank and Stengos (1988) and Frank, Gençay and Stengos (1988). We estimate the largest Lyapunov exponents of these series and investigate the statistical significance of the sign of the exponents using the consistent standard errors. Since the positivity of the Lyapunov exponent in a bounded dissipative nonlinear system is a widely used formal definition of chaos, this approach can be interpreted as a direct test for chaos. ${ }^{1}$

Most of the previous empirical analyses have employed either the correlation dimension or the Lyapunov exponent as a quantity of interest. One problem with the correlation dimension measure, however, is that its sampling properties for chaotic series are unknown, especially when a stochastic term is present in the system (see Barnett et al. (1995, p.306)). Therefore, a formal test cannot be conducted with the correlation dimension. It should also be noted that the well-known BDS test (Brock et al. (1996)) was constructed using the correlation function (which has a direct connection with the correlation dimension). However, the BDS test should be viewed as a test for i.i.d. null against general dependence rather than a direct test for chaos. Our approach involves a type of Lyapunov exponent calculation method called the Jacobian method, which was first proposed by Eckmann and Ruelle (1985). A notable advantage of the Jacobian

\footnotetext{
${ }^{1}$ This definition is introduced by Eckmann and Ruelle (1985).
} 
method over another available method, the direct method developed by Wolf et al. (1985), is its robustness to the presence of (small) stochastic components in the system. ${ }^{2}$ Such a generalization of the notion of chaos is sometimes referred to as noisy chaos as opposed to deterministic chaos. Under this stochastic time series framework, the statistical properties of the Lyapunov exponent estimator can be established by looking at the properties of the nonparametric estimator of the nonlinear autoregressive model. McCaffrey et al. (1992) showed the consistency of a neural networks Lyapunov exponent estimator and Whang and Linton (1999) provided conditions that are sufficient for a kernel estimator to be asymptotically normal at a certain rate.

In principle, any nonparametric derivative estimator can be used for the Jacobian method. However, the neural networks (or neural nets) estimator of the Lyapunov exponent, which was first proposed by Nychka et al. (1992) and Gençay and Dechert (1992), is the most popular method in economics applications. ${ }^{3}$ Theoretically, neural nets are expected to perform better than other approximation methods, especially with high-dimensional models, since the approximation form is not so sensitive to the increasing dimension, at least within the confines of the particular class of functions considered. The reliability of using neural nets in practice was also affirmed in a single-blind controlled competition conducted by Barnett et al. (1997). While the former empirical studies with the neural network estimation only reported the point estimate of the Lyapunov exponent, it is possible to derive the asymptotic distribution of the estimator by employing Whang and Linton's (1999) argument, which was originally used for the Nadaraya-Watson type kernel regression method. In this paper, we test the null hypothesis of the positive Lyapunov exponent, or chaotic hypothesis, using the theoretical asymptotic distribution of the neural net estimator.

In addition to neural networks, we also employ a class of kernel-type regression estimators called local

\footnotetext{
${ }^{2}$ Another advantage of the Jacobian method is that it requires a smaller sample relative to the direct method. These advantages are confirmed by the direct comparison of the two methods in the experiment conducted by Kaashoek and van Dijk (1994).

${ }^{3}$ The examples of economic applications are Dechert and Gençay's (1992) analysis using exchange rates, studies on monetary aggregates by Serletis (1995) and Barnett et al. (1995) and the analysis of stock return series by Abhyankar, Copeland and Wong (1996).
} 
polynomial regression estimators as a nonparametric estimation for the Jacobian method. In fact, the significant advantages of local polynomial regression over the Nadaraya-Watson regression estimator was recently made clear by Fan (1992), Fan and Gijbels (1992) and Ruppert and Wand (1994). First, it reduces the bias of the Nadaraya-Watson estimator. Second, it adapts automatically to the boundary of design points and therefore no boundary modification is needed. Third, it is superior to the Nadaraya-Watson estimator in the context of derivative estimation. As explained in Fan and Gijbels (1996, p.77), the local polynomial of order two, or local quadratic smoother, is preferable over the local linear estimator for first derivative estimation for the same reasons. Therefore, we modify the asymptotic result of Whang and Linton (1999) to establish the asymptotics for the local quadratic estimator of the Lyapunov exponent. We believe reporting the results based on both neural nets and local quadratic smoother is informative since the former is a global nonparametric method while the latter is a local nonparametric method.

The remainder of the paper is organized as follows: An overview of the Lyapunov exponent estimation method and its limit distribution based on the neural networks and the local polynomial regression are provided in Section 2. Section 3 reports the Lyapunov exponent estimates and interprets the results. Section 4 provides the argument on the relation between stochastic trend and chaos. Section 5 discusses possible limitations of our approach with respect to the power of the test. Concluding remarks are made in Section 6. All technical assumptions and proofs are given in the Appendix.

\section{Lyapunov exponent test}

\subsection{Lyapunov exponent of stochastic time series}

In this section, we introduce the method to be used in the empirical analysis. Let $\left\{Y_{t}\right\}_{t=1}^{T}$ be a random scalar sequence generated by the following non-linear autoregressive model

$$
Y_{t}=m\left(Y_{t-1}, \ldots, Y_{t-d}\right)+u_{t},
$$


where $m: \mathbb{R}^{d} \rightarrow \mathbb{R}$ is a non-linear dynamic map and $\left\{u_{t}\right\}_{t=1}^{T}$ is a sequence of martingale differences with $E\left(u_{t} \mid \mathcal{F}_{-\infty}^{t-1}\right)=0$ and $E\left(u_{t}^{2}\right)=\sigma_{t}^{2}<\infty$ where $\mathcal{F}_{s}^{t}$ is the $\sigma$-field generated by $\left(X_{s}, \ldots, X_{t}\right)$ and $X_{t}=$ $\left(Y_{t}, \ldots, Y_{t-d+1}\right)^{\prime} \in \mathbb{R}^{d}$. We also assume $m$ to satisfy a certain smoothness condition and $X_{t}$ to be strictly stationary and to satisfy a class of mixing conditions. ${ }^{4}$ The advantage of this formulation is that we can include both the traditional exogenous business cycle model and the chaotic endogenous business cycle model within the same framework. As mentioned in the introduction, the traditional approach stresses the role of exogenous shocks as a source of aggregate fluctuation with the assumption of a stable steady state. With such a modeling strategy, the stochastic term $u_{t}$ in (1) plays an important role in tracking the business cycle relative to the deterministic part $m$. Furthermore, a stable system with "well-behaved" function $m$ ensures that the impulse response function using linearization around the steady state is a meaningful tool for policy analysis. In contrast, the same system (1) can be noisy chaos if the deterministic system obtained by $\sigma_{t}^{2}=0$ is deterministic chaos, that is, the largest Lyapunov exponent (defined below) computed from the map $m$ is positive.

Let us express the model (1) in terms of a map $F\left(X_{t-1}\right)=\left(m\left(X_{t-1}\right), Y_{t-1}, \ldots, Y_{t-d+1}\right)^{\prime}$ with $U_{t}=$ $\left(u_{t}, 0, \ldots, 0\right)^{\prime}$ such that

$$
X_{t}=F\left(X_{t-1}\right)+U_{t}
$$

and let $J_{t}$ be the Jacobian of the map $F$ in (2) evaluated at $X_{t}$. In this paper, we assume the system (1) has the largest Lyapunov exponent defined by

$$
\lambda \equiv \lim _{M \rightarrow \infty} \frac{1}{2 M} \ln \nu_{1}\left(\mathbf{T}_{M}^{\prime} \mathbf{T}_{M}\right), \quad \mathbf{T}_{M}=\prod_{t=1}^{M} J_{M-t}=J_{M-1} \cdot J_{M-2} \cdots J_{0}
$$

where $\nu_{1}(A)$ is the largest eigenvalue of a matrix $A$. Necessary conditions for the existence of the Lyapunov exponent have been discussed in the literature. (For example, see Nychka et al. (1992, p.406)). It is known

\footnotetext{
${ }^{4}$ See our Appendix, and Whang and Linton (1999) and Shintani and Linton (2000) for the conditions in detail.
} 
that, if $\max \left\{\ln \nu_{1}\left(J_{t}^{\prime} J_{t}\right), 0\right\}$ has a finite first moment with respect to the distribution of $X_{t}$, then the limit in (3) almost surely exists and will be a constant, irrespective of the initial condition.

To obtain the Lyapunov exponent from observational data, Eckmann and Ruelle (1985) and Eckmann et al. (1986) proposed a method, known as the Jacobian method, which is based on nonparametric regression. The basic idea of the Jacobian method is to substitute $m$ in the Jacobian formula by its nonparametric estimator $\widehat{m}$. In other words, it is the sample analogue estimator of (3). It should be noted that we distinguish between the "sample size" $T$ used for estimating Jacobian $\widehat{J}_{t}$ and the "block length" $M$, which is the number of evaluation points used for estimating Lyapunov exponent. Formally, the Lyapunov exponent estimator of $\lambda$ can be obtained by

$$
\widehat{\lambda}_{M}=\frac{1}{2 M} \ln \nu_{1}\left(\widehat{\mathbf{T}}_{M}^{\prime} \widehat{\mathbf{T}}_{M}\right), \quad \widehat{\mathbf{T}}_{M}=\prod_{t=1}^{M} \widehat{J}_{M-t}=\widehat{J}_{M-1} \cdot \widehat{J}_{M-2} \cdots \widehat{J}_{0}
$$

where

$$
\widehat{J}_{t-1}=\left[\begin{array}{ccccc}
\Delta \widehat{m}_{1}\left(X_{t-1}\right) & \Delta \widehat{m}_{2}\left(X_{t-1}\right) & \cdots & \Delta \widehat{m}_{d-1}\left(X_{t-1}\right) & \Delta \widehat{m}_{d}\left(X_{t-1}\right) \\
1 & 0 & \cdots & 0 & 0 \\
0 & 1 & \cdots & 0 & 0 \\
\vdots & \vdots & \ddots & \vdots & \vdots \\
0 & 0 & \cdots & 1 & 0
\end{array}\right]
$$

for $t=0,1, \ldots, M-1$, where $\Delta \widehat{m}_{j}(x)$ is nonparametric estimator of $\Delta m_{j}(x)=\frac{\partial m}{\partial Y_{t-j}}(x)$ for $j=1, \ldots, d$. 


\subsection{Neural networks}

The neural network estimator $\widehat{m}$ can be obtained by minimizing the least square criterion $\sum_{t=1}^{T}\left(Y_{t}-\right.$ $\left.m_{T}\left(X_{t-1}\right)\right)^{2}$, where the neural network sieve $m_{T}: \mathbb{R}^{d} \rightarrow \mathbb{R}$ is an approximation function defined by

$$
m_{T}(x)=\beta_{0}+\sum_{j=1}^{q_{T}} \beta_{j} \psi\left(a_{j}^{\prime} x+b_{j}\right)
$$

where $\psi$ is an activation function and $q_{T}$ is the number of hidden units.

Using the arguments of Whang and Linton (1999), Shintani and Linton (2000) established the asymptotic normality of the neural network estimator $\widehat{\lambda}_{M}$ and provided a consistent standard error $\sqrt{\widehat{\Phi} / M}$ where

$$
\begin{gathered}
\widehat{\Phi}=\sum_{j=-M+1}^{M-1} w\left(j / S_{M}\right) \widehat{\gamma}(j) \text { with } \widehat{\gamma}(j)=\frac{1}{M} \sum_{t=|j|+1}^{M} \widehat{\eta}_{t} \widehat{\eta}_{t-|j|}, \\
\widehat{\eta}_{t}=\widehat{\xi}_{t}-\widehat{\lambda}_{M} \text { with } \widehat{\xi}_{t}=\frac{1}{2} \ln \left(\frac{\nu_{1}\left(\widehat{\mathbf{T}}_{t}^{\prime} \widehat{\mathbf{T}}_{t}\right)}{\nu_{1}\left(\widehat{\mathbf{T}}_{t-1}^{\prime} \widehat{\mathbf{T}}_{t-1}\right)}\right) \text { for } t \geq 2 \text { and } \widehat{\xi}_{1}=\frac{1}{2} \ln \nu_{1}\left(\widehat{\mathbf{T}}_{1}^{\prime} \widehat{\mathbf{T}}_{1}\right),
\end{gathered}
$$

where $w(\cdot)$ and $S_{M}$ denote a kernel function and a lag truncation parameter, respectively.

For the neural network estimator $\widehat{m}$ in the empirical analysis, we use the FUNFITS program developed by Nychka et al. (1996). As an activation function $\psi$, this program uses a type of sigmoid function

$$
\psi(u)=\frac{u(1+|u / 2|)}{2+|u|+u^{2} / 2}
$$

which was also employed by Nychka et al. (1992). The number of hidden units $\left(q_{T}\right)$ in the neural network sieve is selected by minimizing the BIC defined by

$$
B I C=\ln \widehat{\sigma}^{2}+\frac{\ln T}{T}\left[1+q_{T}(d+2)\right]
$$


where $\widehat{\sigma}^{2}=T^{-1} \sum_{t=1}^{T}\left(Y_{t}-\widehat{m}\left(X_{t-1}\right)\right)^{2}$.

For the consistent variance estimator $\widehat{\Phi}$, we employ the QS kernel for $w(\cdot)$ with $S_{M}$ selected by optimal bandwidth selection method developed in Andrews (1991). We report the standard error of the Lyapunov exponent estimate $\widehat{\lambda}_{M}$ and conduct a hypothesis test regarding the sign of $\widehat{\lambda}_{M}$.

\subsection{Local quadratic regression}

The local quadratic estimator at a point $x$ can be obtained by minimizing the weighted least squares criterion $\sum_{t=1}^{T}\left(Y_{t}-\beta_{0}-\beta_{1}^{\prime}\left(X_{t-1}-x\right)-\beta_{2}^{\prime} \operatorname{vech}\left\{\left(X_{t-1}-x\right)\left(X_{t-1}-x\right)^{\prime}\right\}\right)^{2} K_{H}\left(X_{t-1}-x\right)$, where $H$ is the $d \times d$ bandwidth matrix, $K$ is $d$-variate kernel function such that $\int K(u) d u=1$, and $K_{H}(u)=|H|^{-1 / 2} K\left(H^{-1 / 2} u\right)$. For simplicity, in this paper, we use $H=h_{T}^{2} I_{d}$ and a product kernel for $K .^{5}$ The solution is given by

$$
\widehat{\beta}(x)=\left(X_{x}^{\prime} W_{x} X_{x}\right)^{-1} X_{x}^{\prime} W_{x} Y
$$

where

$$
X_{x}=\left[\begin{array}{ccc}
1 & \left(X_{0}-x\right)^{\prime} & \operatorname{vech}^{\prime}\left\{\left(X_{0}-x\right)\left(X_{0}-x\right)^{\prime}\right\} \\
\vdots & \vdots & \vdots \\
1 & \left(X_{T}-x\right)^{\prime} & \operatorname{vech}^{\prime}\left\{\left(X_{T-1}-x\right)\left(X_{T-1}-x\right)^{\prime}\right\}
\end{array}\right]
$$

$Y=\left(Y_{1}, \ldots, Y_{T}\right)^{\prime}$ and $W_{x}=\operatorname{diag}\left\{K_{H}\left(X_{0}-x\right), \ldots, K_{H}\left(X_{T-1}-x\right)\right\}$. The local quadratic estimator of first derivatives $\frac{\partial m}{\partial Y_{t-j}}(x)$ for $j=1, \ldots, d$ are given by

$$
\widehat{\beta}_{1}(x)=\left[\begin{array}{c}
\widehat{\beta}_{11}(x) \\
\vdots \\
\widehat{\beta}_{1 d}(x)
\end{array}\right]=\left[\begin{array}{c}
\Delta \widehat{m}_{1}(x) \\
\vdots \\
\Delta \widehat{m}_{d}(x)
\end{array}\right]
$$

\footnotetext{
${ }^{5}$ A more realistic bandwidth matrix is data dependent, in particular $H=h_{T}^{2} \widehat{\Sigma}^{1 / 2}$, where $\widehat{\Sigma}$ is the sample covariance matrix.
} 
Using the arguments of Whang and Linton (1999), we obtain the limit distribution of local quadratic estimator of the Lyapunov exponent.

Theorem 1. Suppose that assumptions A1 to A\%, which are presented in the Appendix, hold. Then

$$
\sqrt{M}\left(\widehat{\lambda}_{M}-\lambda\right) \stackrel{d}{\rightarrow} N(0, \Phi)
$$

where

$$
\Phi \equiv \lim _{M \rightarrow \infty} \operatorname{var}\left[\frac{1}{\sqrt{M}} \sum_{t=1}^{M} \eta_{t}\right]
$$

where

$$
\eta_{t}=\xi_{t}-\lambda \text { with } \xi_{t}=\frac{1}{2} \ln \left(\frac{\nu_{1}\left(\mathbf{T}_{t}^{\prime} \mathbf{T}_{t}\right)}{\nu_{1}\left(\mathbf{T}_{t-1}^{\prime} \mathbf{T}_{t-1}\right)}\right) \text { for } t \geq 2 \text { and } \xi_{1}=\frac{1}{2} \ln \nu_{1}\left(\mathbf{T}_{1}^{\prime} \mathbf{T}_{1}\right)
$$

Note that the limit distribution of the Lyapunov exponent is identical to the one derived in Whang and Linton (1999) and Shintani and Linton (2000). ${ }^{6}$ Therefore, Theorem 1 implies that the estimator similar to (6) in the previous subsection can be also used to calculate the standard error of the local quadratic Lyapunov exponent estimator. For the local quadratic estimator $\widehat{\beta}_{1}(x)$ in the empirical analysis, we employ the Gaussian kernel. The bandwidth $\left(h_{T}\right)$ is selected by minimizing the residual squares criterion (RSC) given in Fan and Gijbels (1996, p.118), which is known to be a consistent selection method for the local polynomial regression. The kernel function and the method of selecting the lag truncation for the standard error are identical to those used in the neural net case.

\footnotetext{
${ }^{6}$ This is due to the fact that the local Lyapunov exponent term, which does not depend on the estimation method, will be dominant in the limit distribution. See the proof in the Appendix for detail.
} 


\section{Main results}

In this section we estimate the Lyapunov exponent of real output series from various countries. Six industrial countries: Canada, Germany, Italy, Japan, the U.K. and the U.S., that were also examined by Brock (1986), Frank and Stengos (1988) and Frank, Gençay and Stengos (1988), are selected. As real output series, we use quarterly seasonally adjusted real GDP data. All data are taken from International Financial Statistics except Japan where the data are obtained from the Economic Planning Agency's Annual Report on National Accounts. The sample period for each country differs depending on the availability of the data. For Canada, the U.K. and the U.S., the data are available over the period 1957(1)-1999(3), while the data of Germany and Italy were available over the period 1960(1)-1998(4), and the data of Japan are available over the period 1955(2)-1999(1).

The theoretical result in the previous section requires the stationarity of the data. In the analysis, we use the stationary cyclical component obtained by removing the deterministic trend from (the logarithms of) the original series. In the modern business cycle literature, some forms of nonlinear trend are often used instead of a simple linear trend. For this reason, we have employed a nonparametric nonlinear detrending method known as the Hodrick-Prescott (HP) filter, in addition to a traditional linear detrending method. Following the literature in the real business cycle analysis, we set the value of the Hodrick-Prescott smoothing parameter to be 1600, which would eliminate the cycles of period longer than eight years (32 quarters) for quarterly series. ${ }^{7}$ Figure 1 shows the trend components of the U.S. real output series based on both linear trend and nonlinear trend. It can be seen that the deviations from the growth path is emphasized in the linearly detrended case. Another type of trend, known as a stochastic trend, will be discussed in the next section.

Using the deviations from trend rather than using the original data in the analysis is also justifiable from the following argument based on the recent chaos literature. Using a simple asset pricing model, Brock and

\footnotetext{
${ }^{7}$ See Cooley (1995), for example, on the definition of the Hodrick-Prescott filter and its smoothing parameter.
} 
Hommes (1998) showed that it is not the stock price itself but the deviation from a rational expectation fundamental solution that has possibly chaotic dynamics derived from the heterogeneous beliefs of agents. Using a similar argument, we can consider the deviation from the solution of the baseline DSGE model as having resulted from the heterogeneity of the agents. In fact, Durlauf (1993) showed that it is possible to construct a model of business cycles using complementarities among heterogenous industries. Since aggregate output in our analysis can be viewed as a cross-sectional average across different sectors, such an interaction among industries seems to be a possible source of complex dynamics.

To emphasize the possibility of complex behavior generated from a simple structure, the first-order nonlinear models are often used in the literature of chaos in economics. (See also Brock and Hommes (1997, p.1061).) For example, the one lag dynamics can be seen in the overlapping generations model, the neoclassical growth model and in the Euler equation of the intertemporal optimization model. Furthermore, since the existence of chaos in the logistic map depends only on one parameter, the logistic map is one of the most popular and convenient one-dimensional chaotic models used in economics. (See Day (1982), for example.) Table 1 shows the estimated Lyapunov exponents of the output series from various countries based on the assumption of the single-dimensional system. Both estimates based on linear detrended series and HP detrended series are presented. The first column of each detrended series shows the estimates of Lyapunov exponents using the entire sample, which is sometimes referred to as the global Lyapunov exponent estimates. The point estimates show that Lyapunov exponents are negative in many series except for Italy where the local quadratic estimate is negative but the neural network estimate is positive. Furthermore, based on the standard error, most of the negative estimates except for neural net estimates for Germany and the U.K. are significant at the $1 \%$ level, which implies the rejection of the positive Lyapunov exponent hypothesis. ${ }^{8}$ The choice of the detrending method has a greater effect on the estimate than the

\footnotetext{
${ }^{8}$ Our standard error can be also used to test the null hypothesis of negative Lyapunov exponent. For example, the local quadratic estimate for Italy is significantly negative but the neural nets estimate is not significantly positive. However, since the test with power against the chaotic alternative is already available in the literature (BDS test, for example), we focus on the test for chaos using the positive Lyapunov exponent as the null hypothesis.
} 
choice of the nonparametric estimation method. ${ }^{9}$ The absolute values of the negative estimates are larger for the HP detrended series than for the linearly detrended case. This result implies that the system is more stable under a nonlinear trend assumption in the sense that the effect of the shock does not last for a long time.

When the block length $M$ is finite, the Lyapunov exponent is sometimes referred to as the local Lyapunov exponent as opposed to the global Lyapunov exponent. (See Wolff (1992), for example.) As argued by Bailey (1996) and Bailey, Ellner and Nychka (1997), the local Lyapunov exponents provide a more detailed description of a system's dynamics than the global Lyapunov exponent alone in the sense that they can identify the difference in the short-term predictability within different regions of the state space. Since the theoretical development in the previous section uses the fact that the block length $M$ grows at a rate slower than the sample size $T$, we can interpret such an estimate to be more closely related to the local Lyapunov exponent rather than the global one. Following the terminology employed by the former studies, when $M$ is less than the entire sample size, we refer to the estimates as the local Lyapunov exponent estimates. The second and third columns of each detrended series represents the local Lyapunov exponent estimates based on the each blocks from two (non-overlapping) subsamples. On the whole, the estimates do not differ much from the global estimates. However, for Japan, there is some difference in the estimates based on the first and second blocks. The absolute values of the negative estimates are larger for the former half than for latter half period. One interpretation is that Japan is currently in a less stable region than in the past.

The next consideration is to determine if these results remain with the choice of the higher dimension or the higher lag order in the model. In general, it is not unusual for the economic model to have more than one-dimensional (lag) structure. Even if there is only one lag, when the model consists of multivariate simultaneous equations, it is known that the stability of the system can be examined through the Lyapunov

\footnotetext{
${ }^{9}$ In some case, the difference between the results from two nonparametric regression methods is larger in the standard error rather than the Lyapunov exponent.
} 
exponent of one series if additional lags in the estimating equation (1) are introduced. ${ }^{10}$ However, there is a technical difficulty in the nonparametric regression with too many lags when the available sample size is limited. With respect to this "curse of dimensionality" problem, the neural nets are believed to give more reliable results than the local quadratic method. On the other hand, it should be noted that it is the low dimensional chaos that is emphasized in the chaos literature rather than the consideration of the system with a very high dimension. Keeping these issues in mind, we examine the Lyapunov exponents based on the system with moderate dimension $d$.

Table 2 shows the multidimensional result on the Lyapunov exponent estimates from the same series. For the neural network estimates, which have some advantages over the local method, the dimension $(d)$ is selected from 2 to 4 using BIC defined in (7) as well as the number of hidden units $\left(q_{T}\right)$. For the local polynomial estimates, the same dimension is used with bandwidth $\left(h_{T}\right)$ selected using RSC. Clearly, all the point estimates of the global Lyapunov exponents are negative. The neural network estimates for Italy based on HP detrended series is also negative where the opposite result is obtained from the singledimensional case. The local Lyapunov exponent estimates, except for the second half of Japan, are all negative. Surprisingly, based on the standard error, the hypothesis of a positive Lyapunov exponent is significantly rejected for all the local Lyapunov exponents of the HP detrended series. While there is a theoretical advantage of the neural network method in the higher dimensional case, both nonparametric methods provide similar results in terms of the sign of the Lyapunov exponents estimates.

The empirical results in this section provide little evidence to indicate the positivity of the Lyapunov exponent in the international real output series. This conclusion is robust to the choice of nonparametric method as well as to the choice of the dimension of the system. However, it should be noted that a system with a periodic solution also has a negative Lyapunov exponent as well as the stable system. This implies

\footnotetext{
${ }^{10}$ This is justified by the well-known theorem of Takens (1981). This type of lag length is sometimes called the embedding dimension. If the original model consists of $n$ equations, the embedding dimension $d$ must be greater than or equal to $2 n+1$ in order to obtain reliable results.
} 
that the evidence against a positive Lyapunov exponent does not exclude all the class of endogeneity such as the limit cycle case. Nevertheless, focusing on the test for chaos seems to be meaningful because it is such a nonperiodic fluctuation that swayed the theoretical interests of the recent researchers in favor of the endogenous business cycle explanation. ${ }^{11}$ With respect to detrending method, the evidence of the negative Lyapunov exponent is stronger for the HP detrended series where the positivity hypothesis of the Lyapunov exponent is significantly rejected for all the series except for the one-dimensional neural nets result of Italy. Yet, so far, we have not considered the possibility of stochastic trend in the analysis.

\section{Unit roots and chaos}

In the previous section, we have investigated time series properties of economic fluctuation under the assumption of the deterministic trend. If the stochastic trend or the unit root is present in the data, the asymptotic results based on the stationarity assumption cannot be applied. Since the choice between the trend stationarity and difference stationarity is still an unsettled question in macroeconomic time series analysis, it is important to consider both possibilities. ${ }^{12}$ Therefore, in this section, we examine the Lyapunov exponent of time series with a unit root, both theoretically and empirically. If the data is difference stationary, estimation of the Lyapunov exponent defined for the first differenced series may be a reasonable approach to employ.

For theoretical simplicity, we confine our attention to the single dimensional case. For a single dimensional model with a unit root, the system (1) reduces to

$$
Y_{t}=Y_{t-1}+u_{t}
$$

\footnotetext{
${ }^{11}$ For example, it has already been shown that the trade cycle model of Hicks (1950), which is well-known for its periodic fluctuation, can also produce chaotic fluctuation (Hommes (1995)).

${ }^{12}$ In general, the evidence is favorable to difference stationary if a simple linear trend is assumed in the alternative and is favorable to trend stationarity with an assumption of nonlinear trend such as a structural shift.
} 
Let us assume $u_{t} \sim i i d\left(0, \sigma^{2}\right) ;(8)$ is then a random walk process. Since $\Delta m\left(Y_{t-1}\right)=1$ for all $Y_{t-1}$, the Lyapunov exponent for a unit root process (8) is zero by the definition of the single dimensional Lyapunov exponent, $\lambda \equiv \lim _{M \rightarrow \infty} M^{-1} \sum_{t=1}^{M} \ln \left|\Delta m\left(Y_{t-1}\right)\right|$. The first three columns of Table 3 show the empirical results of the Lyapunov exponents estimated from raw data without detrending. While most of the estimates are negative, the sizes of the absolute values are much smaller than those obtained from detrended data presented in Table 1 and 2. The fact that the Lyapunov exponents are close to zero can be considered as an indication of the presence of a unit root. However, at the same time, the standard errors are also very small so that the null hypothesis of zero Lyapunov exponent is rejected for many series. The problem here lies in the fact that the theoretical results in Section 2 are derived under the assumption of stationarity and these results cannot be applied to the nonstationary data such as the one generated from (8). Therefore, in order to examine the subject more closely, we need to discuss the properties of nonparametric regression for the unit root processes.

The analysis of nonparametric regression for the unit root process has not been available until the seminal work of Phillips and Park (1998). Their analysis is built upon the theory for nonlinear transformations of integrated processes developed by Park and Phillips (1999) and their related papers. According to this theory, in the limit, the nonlinear functions of integrated process depend on the local time of a Brownian motion $B$, denoted by $L_{B}(t, s)$, which can be interpreted as the time that $B$ spends in the vicinity of $s$ over the time interval $[0, t]$. Let us denote scaled version local time by $L(t, s)=\sigma^{-2} L_{B}(t, s)$ and the mixed normal distribution by $M N(0, \cdot)$. By extending Phillips and Park's (1998) analysis of the Nadaraya-Watson estimator, we obtain the following limit distribution of local quadratic first derivative estimator for the process $(8) \cdot{ }^{13}$

Theorem 2. Suppose that assumptions B1 to B3, which are presented in the Appendix, hold. Then as

\footnotetext{
${ }^{13}$ To the best of the authors' knowledge, there is no unit root theory applicable to the analysis of neural network estimation. For this reason, we focus in this section on the local quadratic estimation.
} 
$T \rightarrow \infty$,

$$
T^{\frac{1}{4}} h_{T}^{\frac{3}{2}}\{\Delta \widehat{m}(y)-1\} \stackrel{d}{\rightarrow} \begin{cases}M N\left(0, \mu\left\{\left(\frac{K_{1}}{\mu_{2}}\right)^{2}\right\} \sigma^{2} L(1,0)^{-1}\right) & \text { for } y=\text { fixed } \\ M N\left(0, \mu\left\{\left(\frac{K_{1}}{\mu_{2}}\right)^{2}\right\} \sigma^{2} L(1, x)^{-1}\right) & \text { for } y=\sqrt{T} x\end{cases}
$$

where $\mu(F)=\int_{-\infty}^{\infty} F(u) d u, K_{1}=u K(u), K_{2}=u^{2} K(u), \mu_{2}=\mu\left(K_{2}\right)$ and $x=$ constant.

Theorem 2 shows that the local quadratic estimator of the first derivative is $T^{\frac{1}{4}} h_{T}^{\frac{3}{2}}$ consistent and its limit distribution is mixed normal with the mixture variate given by the reciprocal of the local time. Since the parametric estimator of the unit root process is known to be $T$ consistent, nonparametric estimation of the first derivative has slower rate of convergence, which is, in a sense, similar to the stationary case. Using this result, we can derive the asymptotic properties of the Lyapunov exponent estimator and the standard error defined in Section 2.

Corollary 1. Suppose that assumptions in Theorem 2 hold, and $M=T$. Then

$$
\widehat{\lambda}_{M} \stackrel{p}{\rightarrow} 0
$$

In addition, if $S_{M}=o\left(T^{\frac{1}{2}-3 \gamma}\right)$,

$$
\widehat{\Phi} \stackrel{p}{\rightarrow} 0
$$

The first part of Corollary 1 shows the consistency of the Lyapunov exponent estimator even in the nonstationary case. The second part of Corollary 1 shows the variance estimator also converges to zero. This explains the small standard errors obtained in Table 3 and implies that they do not exclude the possibility of the unit root in the data. ${ }^{14}$

\footnotetext{
${ }^{14}$ If we proceed further and derive the limit distribution of $\widehat{\lambda}_{M}$, we may be able to construct a unit root test based on the Lyapunov exponent. This line of approach is pursued by Park and Whang (1999) who have proposed a statistic for testing the random walk hypothesis against the chaotic alternative.
} 
If the process is generated from a unit root process (1) with a stochastic error $u_{t}$, such a process falls in the class of exogenous business cycle models with persistent exogenous shocks. ${ }^{15}$ However, if we introduce the nonlinear model with respect to the first differenced series, similar fluctuation can be produced from chaotic endogenous models. To consider these possibilities, it is reasonable to conduct the analysis of Lyapunov exponent based on the first differenced series. Since the first differenced series satisfies the stationarity assumption, the results in Section 2, are directly applicable. Figure 2 shows the difference between the U.S. business cycle fluctuations defined by the first differenced series and the HP filtered series. It shows that there is more fluctuation in the first difference series than in the HP filtered series. The second three columns of Table 3 shows the Lyapunov exponents estimates based on the first differenced data (or the growth rate data). It is interesting to note that, for all cases, the negative Lyapunov exponent is obtained and the hypothesis of a positive Lyapunov exponent is significantly rejected. The size of the absolute values is much larger than detrended case reported in the previous section. In fact, the Lyapunov exponents are significantly smaller than $-\ln 2(\approx-0.693)$, the value with the system where the difference

in the initial condition will be decreased by half in the next period on average. ${ }^{16}$ If the transformation by the first differencing is appropriate, our results seems to be in favor of an exogenous model with persistent shocks rather than the chaotic model for the first differenced series.

\section{$5 \quad$ Truth in advertising}

We have investigated the possibility of chaos in macroeconomic time series based on the nonparametric Lyapunov exponent estimates. Since computing the standard error provides a direct way to test the hypothesis that the Lyapunov exponent is positive or to construct the confidence intervals, our approach has an advantage over the former empirical studies that only reported the point estimates of the Lyapunov

\footnotetext{
${ }^{15}$ Indeed, assumption of persistency of the technological shocks is often employed in the real business cycle models.

${ }^{16}$ This particular number is closely related to the notion of half-life in the linear stable system. The half-life and the Lyapunov exponent of a linear AR model with AR coefficient 0.5 are 1 and $-\ln 2$, respectively.
} 
exponents. In this respect, we may say that our empirical results has removed at least one obstacle toward the consensus on whether there is chaos for macroeconomic time series.

However, at the same time, it is fair to mention that the several limitations of the analysis leave room for a variety of interpretations of the results. In this section, we list up the several reasons why our results, unfavorable to chaos or complex endogenous fluctuation, can not be considered as a definitive answer.

\subsection{Small sample bias}

The most palpable limitation of our analysis is the available sample size of aggregate economic time series data. Despite the fact that the GDP is the most commonly used series as a measure of business cycles, it is only available quarterly. Compared to the direct method (Wolf et al. (1985)), the Jacobian method, or regression based method, is known to require less observations to obtain reliable Lyapunov exponent estimates. However, since our procedure including the standard error is based on asymptotic theory, the validity of the analysis with sample size less than two hundred observations is still open to question. In contrast to the field of natural science where large number of observation is available for the analysis of chaos, the results based on economic data are likely to suffer more from small sample bias. ${ }^{17}$

We have calculated some formal higher order asymptotic expansions and have found a second order degrees of freedom bias in the Lyapunov exponent estimator (see Linton (1995) for some discussion of second order effects in semiparametric estimation), and this bias is always downward. In our case, the direction of this bias is downward, roughly speaking because the second derivative of the function $x \mapsto \log \left(x^{2}\right)$ is $-1 / x^{2}$ and is always negative. The standard errors can also be downward biased in small samples for essentially the same reason. Numerical evidence also confirms this prediction of small sample bias in the negative direction. In the simulation with a Gaussian AR(1) model (Tables 1 to 3 in Whang and Linton (1999)), when sample sizes are as small as 100 and 200, there are clear downward biases. Such a bias in

\footnotetext{
${ }^{17}$ Such a small sample bias associated with economic data has also been a problem in the analysis of chaos based on the correlation dimension. See Ramsey, Sayers and Rothman (1990) for example.
} 
the negative direction may be a reason of obtaining weak evidence of chaos in our paper.

\subsection{Noise level in noisy chaos}

The Jacobian method used to compute Lyapunov exponents allows stochastic terms to be present in the system. In fact, the asymptotic properties are derived from the nonlinear autoregressive model of the form (1) with an error term referred to as a system noise (or a dynamical noise). In addition, unlike the carefully controlled physical experiment, measurement error and unobserved quantities are more likely to exist in aggregate economic time series. By definition, if the deterministic part of the system generates chaos, it is noisy chaos irrespective of the size of the noise.

The problem here is that, in general, the error term should be extremely small for any procedure to expect good performance on detecting chaos in the deterministic part of the system. The power of the test quickly declines as the noise level increases. Regarding this problem, the simulation method shows how fragile the result can be by changing the size of the stochastic term. In Brock and Sayers (1988, p.84), they mentioned some power of the Lyapunov exponent estimates to detect noisy chaos generated from a tent map with a small measurement noise. Whang and Linton (1999) used the logistic map with a small system noise and obtained standard error for the Lyapunov exponent estimates close to its true standard deviation. However, it should be noted that these results were based on simple one-dimensional map and required extremely small noise. Regarding higher-dimensional maps, Dechert and Gençay (1992) conducted simulations investigating the performance of the neural network Lyapunov exponent estimator applied to the Hénon map with the presence of either system noise or measurement noise. By changing the noise to signal ratio, they have investigated how the estimates deteriorate with noise. The results shows that there is a considerable downward bias when the noise to signal ratio (defined with standard deviations) become as large as 0.1 . Furthermore, more realistic scenario can be considered by conducting simulation with specific economic model that can generate chaos. Indeed, the asset pricing model of Brock 
and Hommes (1998) shows a clear difference between the simulated data with and without the noise in the fundamentals.

These simulation results together suggest that we have to admit that constructing reliable test for general noisy chaos with large measurement noise using currently available techniques is extremely difficult and quite challenging. In this sense, empirical findings of this paper could be considered as an evidence against deterministic chaos or noisy chaos with small noise but should not to be considered as a evidence against noisy chaos in general.

\subsection{Seasonal adjustment and detrending}

Another important issue that needs to be considered is the effect of filtering on the result of our procedure. We estimated Lyapunov exponent using seasonally adjusted real output series. However, judging from the reported results from the former studies, the conclusion can be influenced by conducting a seasonal adjustment. The similar problem also applies to the effect of HP filter we employed as a detrending method. This fact can be seen by comparing the HP detrended results in Table 1 and the results in levels in Table 3 where the number and significance of Lyapunov exponents of the two series are very different. If the certain filtering method does not preserve chaotic property of the series, inappropriate filtering results in less informative estimates. The order of applying two filters may also provide different results. Indeed, such a lack of robustness to the choice of filtering in the analysis of chaos seems to be one of the main reasons why there was little agreement about the existence of chaos in economic data (see Barnett et al. (1995) on the related discussions on the robustness of the analysis).

Related to this issue of filtering is the issue of stochastic trend and first differencing discussed in Section 4. Applying our procedure to the first differenced series is justifiable for the case when the data is a cumulative sum of chaotic series or when the data is such a series plus a stochastic trend. However, it

does not work when it is applied to a chaotic series around a stochastic trend. For example, if there is a 
unit root in the 'fundamental' part of the process (based on the models such as an asset pricing model or a DSGE model), and if chaos arises in the deviation from such a 'fundamental,' as in the Brock and Hommes (1998) model, the procedure employed in Section 4 calculates the Lyapunov exponent of the noise and the first difference of a chaotic process. If we simply plot the typical over-differenced chaotic series (such as logistic map) on a graph as one-dimensional map, the resulting nonlinear mapping is usually pathological and cannot be estimated using nonparametric regression method. This implies that our procedure cannot be used to detect chaos around the stochastic trend.

\subsection{Misspecified nonlinearity and dependence}

The final caveat is on the relation between the Lyapunov exponent and the class of nonlinear time series that is not covered by our theory. For the purpose of deriving asymptotics, dependency of the time series is controlled by a mixing coefficient that decreases at a certain rate (see Assumption A1 in the Appendix).

However, it is well-known that such a mixing condition crucially depends on the nonlinear functional form (see Doukhan (1994) for the relation between mixing and nonlinear AR models). Therefore, if the underlying chaotic process is not mixing, our procedure may not have power to detect the chaos. In addition, recent studies have revealed that the data generated from the deterministic chaotic system, such as the logistic map, can have long range dependence with a certain choice of parameter value (Hall and Wolff (1995) and Guégan (2000)). However, our procedure is designed for the short-memory rather than the long-memory time series.

In contrast to the no consensus of chaos in aggregate data, it is generally agreed that the nonlinearity itself plays an important role in characterizing economics time series. Indeed several classes of nonlinear models, including threshold autoregressive (TAR) models (Tong (1990), Potter (1995), and Hansen (1996)), Markov-Switching models (Hamilton (1989)) and models with duration dependence (Diebold and Rudebusch (1990)) have been very successful in the empirical analysis of business cycles. In order to eval- 
uate the validity of our Lyapunov exponent test for these classes of models, further analysis is required since the interpretation of Lyapunov exponent for these models may be difficult. For example, as shown in Pesaran and Potter (1997), the floor and ceiling structure of Hicks type trade cycle model can be estimated using TAR model. In the analysis of business cycle duration dependence, Diebold and Rudebusch (1990) have introduced the generalized notion of periodicity, including stochastic periodicity and stochastic weak periodicity. Such properties cannot be covered by the system that generates simple periodic fluctuation where the Lyapunov exponent is negative.

\section{Conclusion}

In this paper, we have conducted a nonparametric analysis of the Lyapunov exponent for the purpose of comparing two alternative views of the business cycle - the traditional exogenous and chaotic (or complex) endogenous approaches. The procedure was applied to investigate the real output series from various countries also examined by Brock (1986), Frank and Stengos (1988) and Frank, Gençay and Stengos (1988). The value that we have provided is mostly in the standard error or the confidence intervals based on our asymptotic distribution theory. In this sense, our approach provides a more scientific way of evaluating the data than simply reporting the point estimates. Surprisingly, in many cases, our Lyapunov exponent estimates were negative, although frequently close to zero, and the positivity of the exponent was significantly rejected in the statistical sense. This finding was robust to the choice of nonparametric method as well as to the choice of the dimension of the system. Further theoretical and empirical analyses were also conducted to consider the effect of unit root nonstationarity. The result based on the first differenced series rather than the detrended series again showed significant negative Lyapunov exponents.

A possible interpretation of this result is that the exogenous models are better able to explain business cycle fluctuations than is the chaotic endogenous approach. If this is the case, it supports former studies that did not find evidence of chaos in similar series. Most importantly, the results can be considered as 
an empirical justification of the impulse response analysis that is commonly used among macroeconomists under the framework of the DSGE models.

An alternative interpretation is that we failed to detect chaos because of the limitations of our analysis. As the source of difficulties, we have listed the issues of small sample bias, high noise level in noisy chaos, filtering and the class of nonlinearity of the system. If we consider these issues seriously, our findings cannot exclude all the possibilities of chaos. In this sense, the results reported in this paper may be better considered as one clue rather than a definitive answer to our original question. One remedy to the problem associated with the small sample is to apply our procedure to economic data available with larger number of observations. Since financial time series is usually available daily or even trade by trade, and since a economic theory predicts chaos in the stock market (Brock and Hommes (1998)), analysis of financial market would be of great interest. In addition, many aggregate series other than real GDP series are available monthly. The test based on monetary aggregates and aggregate price is also an important topic. These analyses are currently being conducted by the authors. 


\section{Appendix: Assumptions and proofs}

\section{Assumptions for Theorem 1.}

Assumption A1 (mixing): (1) The process $\left\{Y_{t}\right\}_{t=1}^{T}$ is a strong mixing sequence satisfying $\sum_{j=1}^{\infty} j^{a}\{\alpha(j)\}^{1-2 / v}<$ $\infty$ for some $v>2, a \geq 1-2 / v$, where $\alpha(j)$ is the strong mixing coefficient defined by

$$
\alpha(j)=\sup _{A \in \mathcal{F}_{-\infty}^{0}, B \in \mathcal{F}_{j}^{\infty}}|P(A B)-P(A) P(B)|,
$$

where $\mathcal{F}_{s}^{t}$ is the $\sigma$-field generated by $\left(Y_{s}, \ldots, Y_{t}\right)$.

(2) $E\left|Y_{t}\right|^{\sigma}<\infty$ for some $\sigma \geq v$.

(3) $\alpha(j)$ satisfies $\sum_{T=1}^{\infty} \psi(T)<\infty$, where

$$
\begin{aligned}
\psi(T) & =\frac{T \Lambda_{T}}{r_{T}}\left(\frac{T \tau_{T}}{h_{T} \ln T}\right)^{1 / 4} \alpha\left(r_{T}\right), \\
r_{T} & =\frac{\left(T h_{T}^{d} / \ln T\right)^{1 / 2}}{\tau_{T}}, \quad \Lambda_{T}=\left(\frac{T \tau_{T}^{2}}{h_{T}^{d+2} \ln T}\right)^{d / 2}
\end{aligned}
$$

and $\tau_{T}=\left\{T \ln T(\ln \ln T)^{1+\delta}\right\}^{1 / \sigma}$ for some $0<\delta<1$.

Assumption A2 (density): (1) The probability density function $f(x)$ is bounded and uniformly continuous on $R^{d}$ and $f(x) \leq$ const $<\infty$. (2) The joint density function $f_{X_{t}, X_{t-l}}\left(x, x^{\prime}\right)$ of $X_{t}$ and $X_{t-l}$ satisfies $f_{X_{t}, X_{t-l}}\left(x, x^{\prime}\right) \leq \mathrm{const}<\infty$ for all $l \geq 1$.(3) The conditional density function of $f_{X_{t-1} \mid Y_{t}}(x \mid y)$ of $X_{t-1}$ given $Y_{t}$ exists and is bounded, $f_{X_{t-1} \mid Y_{t}}(x \mid y) \leq$ const $<\infty$. (4) The conditional density function of $f_{\left(X_{t}, X_{t-l}\right) \mid\left(Y_{t}, Y_{t-l}\right)}\left(x, x^{\prime} \mid y, y^{\prime}\right)$ of $\left(X_{t}, X_{t-l}\right)$ given $\left(Y_{t}, Y_{t-l}\right)$ exists and is bounded, i.e.,

$f_{\left(X_{t}, X_{t-l}\right) \mid\left(Y_{t}, Y_{t-l}\right)}\left(x, x^{\prime} \mid y, y^{\prime}\right) \leq \mathrm{const}<\infty$ for all $l \geq 1$. (5) $\inf _{x \in \mathbb{D}} f(x)=$ const $<\infty$, where $\mathbb{D}$ is a compact subset of $\mathbb{R}^{d}$.

Assumption A3 (regression function): (1) $D^{\mathbf{k}} m(x)$ is bounded and uniformly continuous on $\mathbb{R}^{d}$ for $|\mathbf{k}|=3$, where

$$
D^{\mathbf{k}} m(x)=\frac{\partial^{\mid \mathbf{k}} m(x)}{\partial Y_{t-1}^{k_{1}}, \ldots, \partial Y_{t-d}^{k_{d}}},
$$

$\mathbf{k}=\left(k_{1}, \ldots, k_{d}\right)^{\prime} \in \mathbb{R}^{d}$, and a vector norm defined by $|\mathbf{k}| \equiv \sum_{i=1}^{d}\left|k_{i}\right|$. (2) $\sup _{x \in \mathbb{R}^{d}}\left|D^{\mathbf{k}} m(x)\right| \leq \mathrm{const}<\infty$ for $|\mathbf{k}|=3$. (3) $\left|D^{\mathbf{k}} m(x)-D^{\mathbf{k}} m(z)\right| \leq$ const $\times\|x-z\|$ for $|\mathbf{k}|=3$.

Assumption A4 (kernel): (1) $\int\|u\|^{4} K(u) d u<\infty$. (2) $K(u)$ is bounded with compact support. (3) $\left|u^{j} K(u)-v^{j} K(v)\right| \leq \mathrm{const} \times\|u-v\|$ for all $j$ with $0 \leq j \leq 2$.

Assumption A5 (bandwidth):

$$
h_{T}=O\left\{\left(\frac{T}{\ln T}\right)^{-1 /(d+6)}\right\} .
$$

Assumption A6 (extreme value theory): $\max _{1 \leq t \leq M}\left|F_{t-1}\left(J_{M-1}, \ldots, J_{0}\right)\right|=O_{p}\left(M^{\phi}\right)$ for some $\phi \geq 0$, where

$$
F_{t-1}\left(J_{M-1}, \ldots, J_{0}\right)=\frac{\partial \ln \nu_{1}\left(\mathbf{T}_{M}^{\prime} \mathbf{T}_{M}\right)}{\partial \Delta m\left(X_{t-1}\right)} \text { and } \Delta m\left(X_{t-1}\right)=\left(\Delta m_{1, t-1}, \Delta m_{2, t-1}, \ldots, \Delta m_{d, t-1}\right)^{\prime} .
$$


Assumption A7 (block length): $M \rightarrow \infty$ and

$$
M=O\left\{\left(\frac{T}{\ln T}\right)^{4 /\{(d+6)(1+2 \phi)\}}\right\} .
$$

\section{Proof of Theorem 1.}

By Theorem 6 of Masry (1996), under assumptions A1-A5, we have the following uniform convergence rate of the local quadratic first derivative estimator,

$$
\sup _{x \in \mathbb{D}}|\Delta \widehat{m}(x)-\Delta m(x)|=O_{a . s .}\left\{\left(\frac{T}{\ln T}\right)^{-2 /(d+6)}\right\} .
$$

By rearranging terms,

$$
\sqrt{M}\left(\widehat{\lambda}_{M}-\lambda\right)=\sqrt{M}\left(\widehat{\lambda}_{M}-\lambda_{M}\right)+\sqrt{M}\left(\lambda_{M}-\lambda\right),
$$

where $\lambda_{M}$ is the local Lyapunov exponent defined by

$$
\lambda_{M}=\frac{1}{2 M} \ln \nu_{1}\left(\left(\Pi_{t=1}^{M} J_{M-t}\right)^{\prime}\left(\Pi_{t=1}^{M} J_{M-t}\right)\right) .
$$

For the second term, we have

$$
\begin{aligned}
\sqrt{M}\left(\lambda_{M}-\lambda\right) & =\sqrt{M}\left[\frac{1}{2 M} \ln \nu_{1}\left(\left(\Pi_{t=1}^{M} J_{M-t}\right)^{\prime}\left(\Pi_{t=1}^{M} J_{M-t}\right)\right)-\lambda\right] \\
& =\sqrt{M}\left[\frac{1}{2 M} \ln \nu_{1}\left(\mathbf{T}_{M}^{\prime} \mathbf{T}_{M}\right)-\lambda\right] \\
& =\sqrt{M}\left[\frac{1}{2 M} \ln \left(\frac{\nu_{1}\left(\mathbf{T}_{M}^{\prime} \mathbf{T}_{M}\right)}{\nu_{1}\left(\mathbf{T}_{M-1}^{\prime} \mathbf{T}_{M-1}\right)}\right)+\frac{1}{2 M} \ln \nu_{i}\left(\mathbf{T}_{M-1}^{\prime} \mathbf{T}_{M-1}\right)-\lambda\right] \\
& =\sqrt{M}\left[\sum_{k=1}^{M-1} \frac{1}{2 M} \ln \left(\frac{\nu_{1}\left(\mathbf{T}_{M-k+1}^{\prime} \mathbf{T}_{M-k+1}\right)}{\nu_{1}\left(\mathbf{T}_{M-k}^{\prime} \mathbf{T}_{M-k}\right)}\right)+\frac{1}{2 M} \ln \nu_{1}\left(\mathbf{T}_{1}^{\prime} \mathbf{T}_{1}\right)-\lambda\right] \\
& =\sqrt{M}\left[\frac{1}{M} \sum_{k=1}^{M} \xi_{M-k+1}-\lambda\right] \\
& =\frac{1}{\sqrt{M}} \sum_{t=1}^{M}\left[\xi_{t}-\lambda\right] \Rightarrow N(0, \Phi)
\end{aligned}
$$

by the CLT of Herrndorf (1984, Corollary 1) and results of Furstenberg and Kesten (1960, Theorem 3).

For the first term,

$$
\begin{aligned}
\left|\sqrt{M}\left(\widehat{\lambda}-\lambda_{M}\right)\right| & =\frac{1}{2 \sqrt{M}}\left|\ln \nu_{1}\left(\left(\Pi_{t=1}^{M} \widehat{J}_{M-t}\right)^{\prime}\left(\Pi_{t=1}^{M} \widehat{J}_{M-t}\right)\right)-\ln \nu_{1}\left(\left(\Pi_{t=1}^{M} J_{M-t}\right)^{\prime}\left(\Pi_{t=1}^{M} J_{M-t}\right)\right)\right| \\
& =\left|\frac{1}{\sqrt{M}} \sum_{t=1}^{M} F_{t-1}\left(J_{M-1}^{*}, \ldots, J_{0}^{*}\right)^{\prime}\left[\Delta \widehat{m}\left(X_{t-1}\right)-\Delta m\left(X_{t-1}\right)\right]\right|
\end{aligned}
$$




$$
\begin{aligned}
\leq & {[T / \ln T]^{-\frac{2}{6+d}} M^{\frac{1}{2}+\phi}\left[[T / \ln T]^{\frac{2}{6+d}} \sup _{x \in \mathbb{D}}|\Delta \widehat{m}(x)-\Delta m(x)|\right] } \\
& \times M^{-\phi} \max _{1 \leq t \leq M}\left|F_{t-1}\left(J_{M-1}^{*}, \ldots, J_{0}^{*}\right)\right|=o_{p}(1),
\end{aligned}
$$

where the second equality follows from a one-term Taylor expansion

$$
\begin{aligned}
& \ln \nu_{1}\left(\left(\Pi_{t=1}^{M} \widehat{J}_{M-t}\right)^{\prime}\left(\Pi_{t=1}^{M} \widehat{J}_{M-t}\right)\right) \\
= & \ln \nu_{1}\left(\left(\Pi_{t=1}^{M} J_{M-t}\right)^{\prime}\left(\Pi_{t=1}^{M} J_{M-t}\right)\right)+\frac{\partial \ln \nu_{1}\left(\left(\Pi_{t=1}^{M} J_{M-t}^{*}\right)^{\prime}\left(\Pi_{t=1}^{M} J_{M-t}^{*}\right)\right)}{\partial \Delta m_{0}\left(X_{t-1}\right)^{\prime}}\left[\Delta \widehat{m}\left(X_{t-1}\right)-\Delta m\left(X_{t-1}\right)\right] \\
= & \ln \nu_{1}\left(\left(\Pi_{t=1}^{M} J_{M-t}\right)^{\prime}\left(\Pi_{t=1}^{M} J_{M-t}\right)\right)+F_{t-1}\left(J_{M-1}^{*}, \ldots, J_{0}^{*}\right)^{\prime}\left[\Delta \widehat{m}\left(X_{t-1}\right)-\Delta m\left(X_{t-1}\right)\right],
\end{aligned}
$$

where the elements of $J_{t}^{*}$ lie between those of $\widehat{J}_{t}$ and $J_{t}$ for $t=0, \ldots, M-1$. The convergence to zero holds because of $[T / \ln T]^{-\frac{2}{6+d}} M^{\frac{1}{2}+\phi}=O(1)$ from assumption A7, uniform convergence rate of $\Delta \widehat{m}\left(X_{t-1}\right)$ and $M^{-\phi} \max _{1 \leq t \leq M}\left|F_{t-1}\left(J_{M-1}^{*}, \ldots, J_{0}^{*}\right)\right|=O_{p}(1)$ from assumption A6, respectively. The latter can be verified by using the argument given in the proof of Theorem 1 in Whang and Linton (1999).

\section{Assumptions for Theorem 2.}

Assumption B1 (data): (1) $y_{0}=O_{p}(1)(2)\left\{u_{t}\right\}_{j=0}^{\infty}$ is iid $\left(0, \sigma^{2}\right)$ with $E\left(\left|u_{t}\right|^{q}\right)<\infty$, for some $q>4$, and has the distribution absolutely continuous with respect to the Lebesgue measure with characteristic function $\phi(t)$ for which $\lim _{t \rightarrow \infty} t^{r} \phi(t)=0$ for some $r>0$.

Assumption B2 (kernel): (1) $\mu\left(K_{i}\right)=\int u^{i} K(u) d u<\infty \quad$ and $\quad \mu\left(K_{i}^{2}\right)=\int u^{2 i} K(u)^{2} d u<\infty \quad$ for $0 \leq i \leq$ 4. (2) $\mu(K)=\int u K(u) d u=1, \mu\left(K_{2}\right)=\int u^{2} K(u) d u \neq 0, K(x)=K(-x)$ and $\sup K(x)<\infty$.

Assumption B3 (bandwidth):

$$
h_{T}=O\left(T^{-\gamma}\right) \text { for some } 0<\gamma<\frac{1}{10}
$$

\section{Proof of Theorem 2.}

The local quadratic estimator for the univariate case can be written as

$$
\widehat{\beta}(y)=\left[\begin{array}{c}
\widehat{\beta}_{0}(y) \\
\widehat{\beta}_{1}(y) \\
\widehat{\beta}_{2}(y)
\end{array}\right]=\left[\begin{array}{lll}
S_{0}(y) & S_{1}(y) & S_{2}(y) \\
S_{1}(y) & S_{2}(y) & S_{3}(y) \\
S_{2}(y) & S_{3}(y) & S_{4}(y)
\end{array}\right]^{-1}\left[\begin{array}{l}
T_{0}(y) \\
T_{1}(y) \\
T_{2}(y)
\end{array}\right],
$$

where $S_{i}(y)=\sum_{t=1}^{T}\left(Y_{t-1}-y\right)^{i} K_{H}\left(Y_{t-1}-y\right)$ and $T_{i}(y)=\sum_{t=1}^{T}\left(Y_{t-1}-y\right)^{i} K_{H}\left(Y_{t-1}-y\right) Y_{t}$. Substituting $Y_{t}=Y_{t-1}+u_{t}$ in the formula of $\Delta \widehat{m}(y)=\widehat{\beta}_{1}(y)$ yields

$$
T^{\frac{1}{4}} h_{T}^{\frac{3}{2}}\{\Delta \widehat{m}(y)-1\}=\frac{-A_{0}(y)+A_{1}(y)-A_{2}(y)}{B(y)},
$$

where

$$
\begin{aligned}
& A_{0}(y)=\left\{Q_{1}(y) Q_{4}(y)-Q_{2}(y) Q_{3 n}(y)\right\} P_{0}(y) \\
& A_{1}(y)=\left\{Q_{0}(y) Q_{4}(y)-Q_{2}(y)^{2}\right\} P_{1}(y) \\
& A_{2}(y)=\left\{Q_{0}(y) Q_{3}(y)-Q_{1}(y) Q_{2}(y)\right\} P_{2}(y)
\end{aligned}
$$


and

$$
\begin{aligned}
B(y)= & Q_{0}(y) Q_{2}(y) Q_{4}(y)+2 Q_{1}(y) Q_{2}(y) Q_{3}(y) \\
& -Q_{2}(y)^{3}-Q_{0}(y) Q_{3}(y)^{2}-Q_{1}(y)^{2} Q_{4}(y)
\end{aligned}
$$

where

$$
\begin{aligned}
Q_{i}(y) & =\frac{1}{T^{1 / 2} h_{T}} \sum_{t=1}^{T}\left(\frac{Y_{t-1}-y}{h_{T}}\right)^{i} K\left(\frac{Y_{t-1}-y}{h_{T}}\right) \text { and } \\
P_{i}(y) & =\frac{1}{T^{1 / 4} h_{T}^{1 / 2}} \sum_{t=1}^{T}\left(\frac{Y_{t-1}-y}{h_{T}}\right)^{i} K\left(\frac{Y_{t-1}-y}{h_{T}}\right) u_{t} .
\end{aligned}
$$

Let $y$ be a fixed constant. Under assumptions B1-B3, by using the same argument as in Phillips and Park (1998) or Park and Whang (1999), we have

$$
\begin{aligned}
& Q_{0}(y) \stackrel{d}{\rightarrow} \mu(K) L(1,0), \quad Q_{1}(y) \stackrel{p}{\rightarrow} 0 \\
& Q_{2}(y) \stackrel{d}{\rightarrow} \mu\left(K_{2}\right) L(1,0), \quad Q_{3}(y) \stackrel{p}{\rightarrow} 0 \\
& Q_{4}(y) \stackrel{d}{\rightarrow} \mu\left(K_{4}\right) L(1,0)
\end{aligned}
$$

and

$$
P_{i}(y) \stackrel{d}{\rightarrow}\left\{\mu\left(K_{i}^{2}\right) \sigma^{2} L(1,0)\right\}^{1 / 2} V(1) \quad \text { for } 0 \leq j \leq 2,
$$

where $V$ is a standard Brownian motion independent of $B$. Therefore, the first and third elements of (9) are negligible since

$$
A_{0}(y), A_{2}(y) \stackrel{p}{\rightarrow} 0
$$

and

$$
B(y)=Q_{0}(y) Q_{2}(y) Q_{4}(y)-Q_{2}(y)^{3}+o_{p}(1) \stackrel{d}{\rightarrow} \mu\left(K_{2}\right)\left\{\mu\left(K_{4}\right)-\mu\left(K_{2}\right)^{2}\right\} L(1,0)^{3}>0 .
$$

Finally,

$$
\frac{A_{1}(y)}{B(y)} \stackrel{d}{\rightarrow} \frac{\left\{\mu\left(K_{4}\right)-\mu\left(K_{2}\right)^{2}\right\} L(1,0)^{2}\left\{\mu\left(K_{1}^{2}\right) \sigma^{2} L(1,0)\right\}^{1 / 2} V(1)}{\mu\left(K_{2}\right)\left\{\mu\left(K_{4}\right)-\mu\left(K_{2}\right)^{2}\right\} L(1,0)^{3}}=\left\{\frac{\mu\left(K_{1}^{2}\right) \sigma^{2}}{\mu\left(K_{2}\right)^{2} L(1,0)}\right\}^{1 / 2} V(1),
$$

which yields required result. The case with $y=\sqrt{T} x$ can be proved by using the same argument and replacing $L(1,0)$ by $L(1, x)$.

\section{Proof of Corollary 1.}

(i) From the definition, the Lyapunov exponent estimator can be rewritten as

$$
\begin{aligned}
\widehat{\lambda}_{M} & =\frac{1}{T} \sum_{t=1}^{T} \ln \left|\Delta \widehat{m}\left(Y_{t-1}\right)\right| \\
& =\int_{0}^{1}\{\ln |\Delta \widehat{m}(\sqrt{T} B(r))|\} d r+o_{p}(1) \\
& =\int_{0}^{1}\{\ln |1+(\Delta \widehat{m}(\sqrt{T} B(r))-1)|\} d r+o_{p}(1)
\end{aligned}
$$


where the second equality holds by the strong approximation result. The required result follows from $\Delta \widehat{m}(\sqrt{T} x) \stackrel{p}{\rightarrow} 1$ from Theorem 2 .

(ii) Using the result by Park and Whang (1999), $\widehat{\lambda}_{M}=O_{p}\left(T^{-\frac{1}{2}} h_{T}^{-3}\right)$. In addition $\left\{\Delta \widehat{m}\left(Y_{t-1}\right)-1\right\}=$ $O_{p}\left(T^{-\frac{1}{4}} h_{T}^{-\frac{3}{2}}\right)$ from Theorem 2, which implies $\ln \left|\Delta \widehat{m}\left(Y_{t-1}\right)\right|=O_{p}\left(T^{-\frac{1}{4}} h_{T}^{-\frac{3}{2}}\right)$.

$$
\begin{aligned}
\widehat{\eta}_{t}= & \ln \left|\Delta \widehat{m}\left(Y_{t-1}\right)\right|-\widehat{\lambda}_{M} \\
= & O_{p}\left(T^{-\frac{1}{4}} h_{T}^{-\frac{3}{2}}\right)=O_{p}\left(T^{-\frac{1}{4}+\frac{3}{2} \gamma}\right) \quad \text { and } \\
& \widehat{\gamma}(j)=O_{p}\left(T^{-\frac{1}{2}+3 \gamma}\right) .
\end{aligned}
$$

Therefore

$$
\widehat{\Phi}=O\left(S_{M}\right) \times O_{p}\left(T^{-\frac{1}{2}+3 \gamma}\right)=o\left(T^{\frac{1}{2}-3 \gamma}\right) \times O_{p}\left(T^{-\frac{1}{2}+3 \gamma}\right)=o_{p}(1)
$$

\section{References}

Abhyankar, A., L. S. Copeland and W. Wong, "Uncovering Nonlinear Structure in Real-Time StockMarket Indexes: The S\&P 500, the DAX, the Nikkei 225, and the FTSE-100," Journal of Business and Economic Statistics 15 (1997), 1-14.

Andrews, D. W. K., "Heteroskedasticity and Autocorrelation Consistent Covariance Matrix Estimation," Econometrica 59 (1991), 817-858.

Bailey, B. A., "Local Lyapunov Exponents: Predictability Depends on Where You Are," in W. A. Barnett, A. P. Kirman and M. Salmon, eds., Nonlinear Dynamics and Economics (Cambridge: Cambridge University Press) 1996, 345-359.

Bailey, B. A., S. Ellner and D. W. Nychka, "Chaos with Confidence: Asymptotics and Applications of Local Lyapunov Exponents," Fields Institute Communications 11 (1997), 115-133.

Barnett, W. A. and P. Chen, "The Aggregation-Theoretic Monetary Aggregates Are Chaotic and Have Strange Attractors: An Econometric Application of Mathematical Chaos," in W. A. Barnett, E. R. Berndt and H. White, eds., Dynamic Econometric Modeling (Cambridge: Cambridge University Press, 1988), 199-245.

Barnett, W. A., A. R. Gallant, M. J. Hinich, J. A. Jungeilges, D. T. Kaplan and M. J. Jensen, "Robustness of Nonlinearity and Chaos Tests to Measurement Error, Inference Method, and Sample Size," Journal of Economic Behavior and Organization 27 (1995), 301-320.

Barnett, W. A., A. R. Gallant, M. J. Hinich, J. A. Jungeilges, D. T. Kaplan and M. J. Jensen, "A SingleBlind Controlled Competition Among Tests for Nonlinearity and Chaos," Journal of Econometrics 82 (1997), 157-192.

Benhabib, J. and K. Nishimura, "The Hopf Bifurcation and the Existence and Stability of Closed Orbits in Multi-Sector Models of Optimal Economic Growth," Journal of Economic Theory 21 (1979), 421-444.

Brock, W. A., "Distinguishing Random and Deterministic Systems: Abridged Version," Journal of Economic Theory 40 (1986), 168-195. 
Brock, W. A., W. D. Dechert, J. A. Scheinkman and B. LeBaron, "A Test for Independence Based on the Correlation Dimension," Econometric Reviews 15 (1996), 197-235.

Brock, W. A. and C. H. Hommes, "A Rational Route to Randomness," Econometrica 65 (1997), 10591095.

Brock, W. A. and C. H. Hommes, "Heterogeneous Beliefs and Routes to Chaos in a Simple Asset Pricing Model," Journal of Economic Dynamics and Control 22 (1998), 1235-1274.

Brock, W. A. and C. L. Sayers, "Is the Business Cycle Characterized by Deterministic Chaos?" Journal of Monetary Economics 22 (1988), 71-90.

Campbell, J. Y., "Inspecting the Mechanism: An Analytical Approach to the Stochastic Growth Model," Journal of Monetary Economics 33 (1994), 463-506.

Cooley, T. F., Frontiers of Business Cycle Research (Princeton: Princeton University Press, 1995).

Day, R. H., "Irregular Growth Cycles," American Economic Review 72 (1982), 406-414.

Dechert, W. D. and R. Gençay, "Lyapunov Exponents As a Nonparametric Diagnostic for Stability Analysis," Journal of Applied Econometrics 7 (1992), S41-S60.

DeCoster, G. P. and D. W. Mitchell, "Nonlinear Monetary Dynamics," Journal of Business and Economic Statistics 9 (1991), 455-462.

Diebold, F. X. and G. D. Rudebusch, "A Nonparametric Investigation of Duration Dependence In the American Business Cycles," Journal of Political Economy 98 (1990), 596-616.

Doukhan, P., Mixing: Properties and Examples (New York: Springer, 1994).

Durlauf, S. N., "Nonergodic Economic Growth," Review of Economic Studies 60 (1993), 349-366.

Eckmann, J. P., S. O. Kamphorst, D. Ruelle and S. Ciliberto, "Liapunov Exponents from Time Series," Physical Review A 34 (1986), 4971-4979.

Eckmann, J. P. and D. Ruelle, "Ergodic Theory of Chaos and Strange Attractors," Reviews of Modern Physics 57 (1985), 617-656.

Fan, J., "Design-Adaptive Nonparametric Regression," Journal of the American Statistical Association 87 (1992), 998-1004.

Fan, J. and I. Gijbels, "Variable Bandwidth and Local Linear Regression Smoothers," Annals of Statistics 20 (1992), 2008-2036.

Fan, J. and I. Gijbels, Local Polynomial Modelling and Its Applications (London: Chapman and Hall, 1996).

Frank, M., R. Gençay and T. Stengos, "International Chaos?" European Economic Review 32 (1988), 1569-1584.

Frank, M. Z. and T. Stengos, "Some Evidence Concerning Macroeconomic Chaos," Journal of Monetary Economics 22 (1988), 423-438.

Furstenberg, H. and H. Kesten, "Products of Random Matrices," Annals of Mathematical Statistics 31 (1960), 457-469. 
Gençay, R. and W. D. Dechert, "An Algorithm for the n Lyapunov Exponents of an n-Dimensional Unknown Dynamical System," Physica D 59 (1992), 142-157.

Grandmont, J.-M., "On Endogenous Competitive Business Cycles," Econometrica 53 (1985), 995-1045.

Guégan, D., "Stochasticity and Deterministic Chaotic Systems," mimeo, University of Reims, France, 2000.

Hall, P. and R. C. L. Wolff, "On the Strength of Dependence of a Time Series Generated By Chaotic Map," Journal of Time Series Analysis 16 (1995), 571-583.

Hamilton, J., "A New Approach to the Economic Analysis of Nonstationary Time Series and the Business Cycle," Econometrica 57 (1989), 357-384.

Hansen, B. E., "Inference When a Nuisance Parameter is not Identified Under the Null Hypothesis," Econometrica 64 (1996), 413-430.

Herrndorf, N., "An Invariance Principle for Weakly Dependent Sequences of Random Variables," Annals of Probability 12 (1984), 141-153.

Hicks, J. R., A Contribution to the Theory of the Trade Cycle (Oxford: Oxford University Press, 1950).

Hommes, C. H., "A Reconsideration of Hicks' Non-Linear Trade Cycle Model," Structural Change and Economic Dynamics 6 (1995), 435-459.

Kaashoek, J. F. and H. K. v. Dijk, "A Neural Network Applied to the Calculation of Lyapunov Exponents," Econometric Reviews 13 (1994), 123-137.

Kaldor, N., "A Model of the Trade Cycle," Economic Journal 50 (1940), 78-92.

Linton, O., "Second Order Approximation in the Partially Linear Regression Model," Econometrica 63 (1995), 1079-1112.

Masry, E., "Multivariate Local Polynomial Regression for Time Series: Uniform Strong Consistency and Rates," Journal of Time Series Analysis 17 (1996), 571-599.

McCaffrey, D. F., S. Ellner, A. R. Gallant and D. W. Nychka, "Estimating the Lyapunov Exponent of a Chaotic System with Nonparametric Regression," Journal of the American Statistical Association 87(1992), 682-695.

Nychka, D., B. Bailey, S. Ellner, P. Haaland and M. O'Connell, "FUNFITS: Data Analysis and Statistical Tools for Estimating Functions," North Carolina Institute of Statistics Mimeoseries No. 2289, 1996.

Nychka, D., S. Ellner, D. McCaffrey, and A. R. Gallant, "Finding Chaos in Noisy Systems," Journal of the Royal Statistical Society B 54 (1992), 399-426.

Park, J. Y. and P. C. B. Phillips, "Asymptotics for Nonlinear Transformations of Integrated Time Series," Econometric Theory 15 (1999), 269-298.

Park, J. Y. and Y.-J. Whang, "Random Walk or Chaos: A Formal Test on the Lyapunov Exponent," mimeo, Seoul National University and Ewha University, 1999.

Pesaran, M. H. and S. M. Potter, "A Floor and Ceiling Model of US Output," Journal of Economic Dynamics and Control 21 (1997), 661-695. 
Phillips, P. C. B. and J. Y. Park, "Nonstationary Density Estimation and Kernel Autoregression," Cowles Foundation Discussion Paper No. 1181, 1998.

Potter, S. M., "A Nonlinear Approach to US GNP," Journal of Applied Econometrics 10 (1995), 109-125.

Ramsey, J. B., C. L. Sayers and P. Rothman, "The Statistical Properties of Dimension Calculations Using Small Data Sets: Some Economic Applications," International Economic Review 31 (1990), 991-1020.

Ruppert, D. and M. P. Wand, "Multivariate Locally Weighted Least Squares Regression," Annals of Statistics 22 (1994), 1346-1370.

Serletis, A., "Random Walks, Breaking Trend Functions, and the Chaotic Structure of the Velocity of Money," Journal of Business and Economic Statistics 13 (1995), 453-458.

Shintani, M. and O. Linton, "Nonparametric Neural Network Estimation of Lyapunov Exponents and Direct Test for Chaos," mimeo, Vanderbilt University and London School of Economics, 2000.

Sims, C. A., "Macroeconomics and Reality," Econometrica 48 (1980), 1-48.

Slutzky, E., "The Summation of Random Causes as the Source of Cyclical Processes," in Problems of Economic Conditions (Moscow: Conjuncture Institute, 1927)[also published in Econometrica, 1937, 105-146].

Takens, F., "Detecting Strange Attractors in Turbulence," in D. Rand and L. S. Young, eds., Dynamical Systems and Turbulence (Berlin: Springer-Verlag, 1981), 366-381.

Tong, H., Non-linear Time Series: A Dynamical Systems Approach (Oxford: Oxford University Press, 1990).

Whang, Y.-J. and O. Linton, "The Asymptotic Distribution of Nonparametric Estimates of the Lyapunov Exponent for Stochastic Time Series," Journal of Econometrics 91(1999), 1-42.

Wolf, A., J. B. Swift, H. L. Swinney and J. A. Vastano, "Determining Lyapunov Exponents from a Time Series," Physica D 16 (1985), 285-317.

Wolff, R. C. L., "Local Lyapunov Exponents: Looking Closely at Chaos," Journal of the Royal Statistical Society B 54 (1992), 353-371. 
Table 1

Lyapunov Exponent Estimates

(Univariate Results)

\begin{tabular}{|c|c|c|c|c|c|c|c|}
\hline \multirow{6}{*}{$\begin{array}{l}\begin{array}{l}\text { Country } \\
\text { (Sample Size) }\end{array} \\
1 . \text { Canada } \\
(\mathrm{T}=170)\end{array}$} & \multirow{4}{*}{$\begin{array}{c}\text { Method } \\
\text { NN }\end{array}$} & \multicolumn{3}{|c|}{ Linear Detrend } & \multicolumn{3}{|c|}{ HP Detrend } \\
\hline & & \multirow{3}{*}{$\begin{array}{c}\text { Global } \\
-0.031 \\
(0.002)\end{array}$} & \multicolumn{2}{|c|}{ Local } & \multirow{3}{*}{$\begin{array}{c}\text { Global } \\
-0.213 \\
(0.005)\end{array}$} & \multicolumn{2}{|c|}{ Local } \\
\hline & & & -0.030 & -0.031 & & -0.210 & -0.216 \\
\hline & & & $(0.009)$ & $(0.008)$ & & $(0.005)$ & $(0.006)$ \\
\hline & LQ & -0.055 & -0.055 & -0.056 & -0.205 & -0.207 & -0.202 \\
\hline & & $(0.018)$ & $(0.025)$ & $(0.013)$ & $(0.004)$ & $(0.004)$ & $(0.005)$ \\
\hline \multirow{4}{*}{$\begin{array}{l}\text { 2. Germany } \\
(\mathrm{T}=155)\end{array}$} & $\overline{\mathrm{NN}}$ & -0.092 & -0.162 & -0.023 & -0.451 & -0.482 & -0.422 \\
\hline & & $(0.053)$ & $(0.048)$ & $(0.108)$ & $(0.096)$ & $(0.149)$ & $(0.131)$ \\
\hline & LQ & -0.131 & -0.133 & -0.129 & -0.363 & -0.370 & -0.356 \\
\hline & & $(0.038)$ & $(0.057)$ & $(0.049)$ & $(0.019)$ & $(0.028)$ & $(0.027)$ \\
\hline \multirow{4}{*}{$\begin{array}{l}\text { 3. Italy } \\
(\mathrm{T}=155)\end{array}$} & $\mathrm{NN}$ & -0.054 & -0.058 & -0.050 & 0.092 & 0.173 & 0.013 \\
\hline & & $(0.022)$ & $(0.021)$ & $(0.037)$ & $(0.077)$ & $(0.128)$ & $(0.081)$ \\
\hline & LQ & -0.037 & -0.045 & -0.028 & -0.220 & -0.244 & -0.197 \\
\hline & & $(0.007)$ & $(0.010)$ & $(0.001)$ & $(0.020)$ & $(0.038)$ & $(0.003)$ \\
\hline \multirow{4}{*}{$\begin{array}{l}\text { 4. Japan } \\
(\mathrm{T}=175)\end{array}$} & $\overline{\mathrm{NN}}$ & -0.011 & -0.020 & -0.001 & -0.356 & -0.487 & -0.215 \\
\hline & & $(0.003)$ & $(0.005)$ & $(0.006)$ & $(0.056)$ & $(0.070)$ & $(0.063)$ \\
\hline & LQ & -0.008 & -0.011 & -0.004 & -0.449 & -0.594 & -0.287 \\
\hline & & $(0.001)$ & $(0.002)$ & $(0.002)$ & $(0.059)$ & $(0.091)$ & $(0.045)$ \\
\hline \multirow{4}{*}{$\begin{array}{l}\text { 5. U.K. } \\
(\mathrm{T}=170)\end{array}$} & $\mathrm{NN}$ & -0.042 & -0.039 & -0.045 & -0.272 & -0.267 & -0.277 \\
\hline & & $(0.047)$ & $(0.078)$ & $(0.051)$ & $(0.037)$ & $(0.045)$ & $(0.059)$ \\
\hline & LQ & -0.089 & -0.110 & -0.067 & -0.348 & -0.363 & -0.332 \\
\hline & & $(0.016)$ & $(0.018)$ & $(0.017)$ & $(0.038)$ & $(0.059)$ & $(0.053)$ \\
\hline \multirow{4}{*}{$\begin{array}{l}\text { 6. U.S. } \\
(\mathrm{T}=170)\end{array}$} & $\overline{\mathrm{NN}}$ & -0.107 & -0.155 & -0.059 & -0.193 & -0.196 & -0.190 \\
\hline & & (0.037) & $(0.069)$ & $(0.019)$ & $(0.003)$ & $(0.004)$ & $(0.005)$ \\
\hline & LQ & -0.048 & -0.063 & -0.033 & -0.189 & -0.191 & -0.187 \\
\hline & & $(0.008)$ & $(0.009)$ & $(0.005)$ & $(0.003)$ & $(0.005)$ & $(0.005)$ \\
\hline
\end{tabular}

Note: Standard errors $(\sqrt{\widehat{\Phi} / M})$ are in the parentheses. Both estimates using neural networks (NN) and local quadratic smoother (LQ) are presented. The global estimates are based on the entire sample, while the local estimates are based on two (non-overlapping) blocks. QS kernel estimator with optimal bandwidth (Andrews (1991)) is used for the standard errors. The $1 \%$ level critical value for the null hypothesis of $\lambda>0$ is -2.326 , which should be compared with $\widehat{\lambda}_{M} / \sqrt{\widehat{\Phi} / M}$. Bold font indicates that this null hypothesis could not be rejected at the $1 \%$ level of significance. 
Table 2

Lyapunov Exponent Estimates

(Multidimensional Results)

\begin{tabular}{|c|c|c|c|c|c|c|c|}
\hline \multirow{6}{*}{$\begin{array}{l}\begin{array}{l}\text { Country } \\
\text { (Dimension) }\end{array} \\
1 . \text { Canada } \\
(\mathrm{d}=2)\end{array}$} & \multirow{4}{*}{$\begin{array}{c}\text { Method } \\
\text { NN }\end{array}$} & \multicolumn{3}{|c|}{ Linear Detrend } & \multicolumn{3}{|c|}{ HP Detrend } \\
\hline & & \multirow{3}{*}{$\begin{array}{l}\text { Global } \\
-0.052 \\
(0.021)\end{array}$} & \multicolumn{2}{|c|}{ Local } & \multirow{3}{*}{$\begin{array}{c}\text { Global } \\
-0.364 \\
(0.009)\end{array}$} & \multicolumn{2}{|c|}{ Local } \\
\hline & & & -0.047 & -0.046 & & -0.354 & -0.361 \\
\hline & & & $(0.027)$ & $(0.020)$ & & $(0.014)$ & $(0.015)$ \\
\hline & LQ & -0.037 & -0.024 & -0.041 & -0.389 & -0.375 & -0.385 \\
\hline & & $(0.008)$ & $(0.010)$ & $(0.012)$ & $(0.017)$ & $(0.024)$ & $(0.027)$ \\
\hline \multirow{4}{*}{$\begin{array}{l}\text { 2. Germany } \\
(\mathrm{d}=2)\end{array}$} & NN & -0.114 & -0.124 & -0.099 & -0.316 & -0.315 & -0.312 \\
\hline & & $(0.029)$ & $(0.039)$ & $(0.040)$ & (0.018) & $(0.026)$ & (0.031) \\
\hline & LQ & -0.068 & -0.065 & -0.069 & -0.272 & -0.265 & -0.276 \\
\hline & & $(0.009)$ & $(0.011)$ & $(0.011)$ & $(0.012)$ & $(0.017)$ & $(0.016)$ \\
\hline \multirow{4}{*}{$\begin{array}{l}\text { 3. Italy } \\
(\mathrm{d}=3)\end{array}$} & $\mathrm{NN}$ & -0.129 & -0.188 & -0.041 & -0.254 & -0.253 & -0.245 \\
\hline & & $(0.051)$ & $(0.059)$ & $(0.036)$ & $(0.007)$ & $(0.016)$ & (0.018) \\
\hline & LQ & -0.133 & -0.160 & -0.079 & -0.189 & -0.171 & -0.184 \\
\hline & & $(0.033)$ & $(0.037)$ & $(0.040)$ & $(0.015)$ & $(0.035)$ & $(0.028)$ \\
\hline \multirow{4}{*}{$\begin{array}{l}\text { 4. Japan } \\
(\mathrm{d}=4)\end{array}$} & $\overline{\mathrm{NN}}$ & -0.013 & -0.030 & 0.027 & -0.184 & -0.217 & -0.147 \\
\hline & & $(0.021)$ & $(0.029)$ & $(0.019)$ & $(0.016)$ & $(0.021)$ & $(0.026)$ \\
\hline & LQ & -0.034 & -0.042 & 0.003 & -0.171 & -0.162 & -0.168 \\
\hline & & $(0.020)$ & $(0.030)$ & $(0.024)$ & $(0.025)$ & $(0.033)$ & $(0.040)$ \\
\hline \multirow{4}{*}{$\begin{array}{l}\text { 5. U.K. } \\
(\mathrm{d}=4)\end{array}$} & $\overline{\mathrm{NN}}$ & -0.160 & -0.178 & -0.108 & -0.207 & -0.196 & -0.194 \\
\hline & & $(0.030)$ & $(0.041)$ & $(0.037)$ & $(0.021)$ & $(0.029)$ & $(0.035)$ \\
\hline & LQ & -0.094 & -0.085 & -0.087 & -0.220 & -0.217 & -0.208 \\
\hline & & $(0.020)$ & $(0.033)$ & $(0.023)$ & $(0.031)$ & $(0.048)$ & $(0.044)$ \\
\hline \multirow{4}{*}{$\begin{array}{l}\text { 6. U.S. } \\
(\mathrm{d}=2 \text { for Linear, } \\
\mathrm{d}=3 \text { for } \mathrm{HP})\end{array}$} & NN & -0.089 & -0.131 & -0.036 & -0.180 & -0.175 & -0.180 \\
\hline & & $(0.022)$ & (0.027) & (0.011) & (0.009) & $(0.018)$ & $(0.013)$ \\
\hline & LQ & -0.100 & -0.125 & -0.067 & -0.208 & -0.201 & -0.200 \\
\hline & & $(0.013)$ & $(0.022)$ & (0.009) & $(0.016)$ & $(0.026)$ & $(0.028)$ \\
\hline
\end{tabular}

Note: See note of Table 1. 
Table 3

Lyapunov Exponent Estimates Based on Levels and First Differences Specifications

\begin{tabular}{|c|c|c|c|c|c|c|c|}
\hline \multirow[t]{2}{*}{ Country } & \multirow[t]{2}{*}{ Method } & \multicolumn{3}{|c|}{ Levels } & \multicolumn{3}{|c|}{ First Differences } \\
\hline & & Global & \multicolumn{2}{|c|}{ Local } & \multirow{2}{*}{ Global } & \multicolumn{2}{|c|}{ Local } \\
\hline \multirow[t]{4}{*}{ 1. Canada } & NN & -0.013 & -0.004 & -0.022 & & -1.555 & -1.365 \\
\hline & & $(0.004)$ & $(0.004)$ & $(0.003)$ & $(0.032)$ & $(0.042)$ & $(0.043)$ \\
\hline & LQ & -0.002 & 0.002 & -0.007 & -1.344 & -1.355 & -1.332 \\
\hline & & $(0.003)$ & $(0.002)$ & $(0.000)$ & $(0.028)$ & $(0.031)$ & $(0.049)$ \\
\hline \multirow[t]{4}{*}{ 2. Germany } & $\overline{\mathrm{NN}}$ & -0.034 & -0.027 & -0.038 & -4.121 & -4.292 & -3.949 \\
\hline & & $(0.012)$ & $(0.016)$ & $(0.026)$ & $(0.127)$ & $(0.149)$ & $(0.164)$ \\
\hline & LQ & -0.007 & -0.012 & -0.003 & -2.450 & -2.507 & -2.393 \\
\hline & & $(0.003)$ & $(0.002)$ & $(0.000)$ & $(0.122)$ & $(0.193)$ & $(0.146)$ \\
\hline \multirow[t]{4}{*}{ 3. Italy } & $\overline{\mathrm{NN}}$ & -0.023 & -0.013 & $\begin{array}{l}-0.033 \\
\end{array}$ & -1.370 & -0.856 & -1.884 \\
\hline & & $(0.003)$ & $(0.010)$ & $(0.001)$ & $(0.158)$ & $(0.233)$ & $(0.171)$ \\
\hline & LQ & -0.011 & -0.009 & -0.012 & -0.985 & -0.924 & -1.046 \\
\hline & & $(0.001)$ & $(0.002)$ & $(0.000)$ & $(0.073)$ & $(0.105)$ & $(0.098)$ \\
\hline \multirow[t]{4}{*}{ 4. Japan } & $\mathrm{NN}$ & -0.020 & -0.009 & -0.030 & -1.324 & -1.000 & -1.647 \\
\hline & & $(0.002)$ & $(0.004)$ & $(0.002)$ & $(0.118)$ & $(0.127)$ & $(0.172)$ \\
\hline & LQ & -0.009 & -0.004 & -0.014 & -1.283 & -1.141 & -1.426 \\
\hline & & $(0.003)$ & $(0.003)$ & $(0.000)$ & $(0.082)$ & $(0.101)$ & $(0.142)$ \\
\hline \multirow[t]{4}{*}{ 5. U.K. } & NN & -0.010 & -0.010 & -0.010 & -4.485 & -4.378 & -4.592 \\
\hline & & $(0.002)$ & $(0.010)$ & $(0.007)$ & $(0.089)$ & $(0.136)$ & $(0.130)$ \\
\hline & LQ & -0.001 & -0.004 & 0.002 & -2.227 & -2.186 & -2.270 \\
\hline & & $(0.001)$ & $(0.000)$ & $(0.000)$ & $(0.061)$ & $(0.088)$ & $(0.087)$ \\
\hline \multirow[t]{4}{*}{ 6. U.S. } & $\mathrm{NN}$ & -0.004 & -0.007 & -0.002 & -1.076 & -1.098 & -1.054 \\
\hline & & $(0.002)$ & $(0.003)$ & $(0.002)$ & $(0.011)$ & $(0.018)$ & $(0.011)$ \\
\hline & LQ & 0.000 & -0.003 & 0.003 & -1.106 & -1.100 & -1.114 \\
\hline & & $(0.002)$ & $(0.001)$ & $(0.001)$ & $(0.020)$ & $(0.016)$ & $(0.038)$ \\
\hline
\end{tabular}

Note: See note of Table 1. 
Figure 1

US Real Dutput and Trend

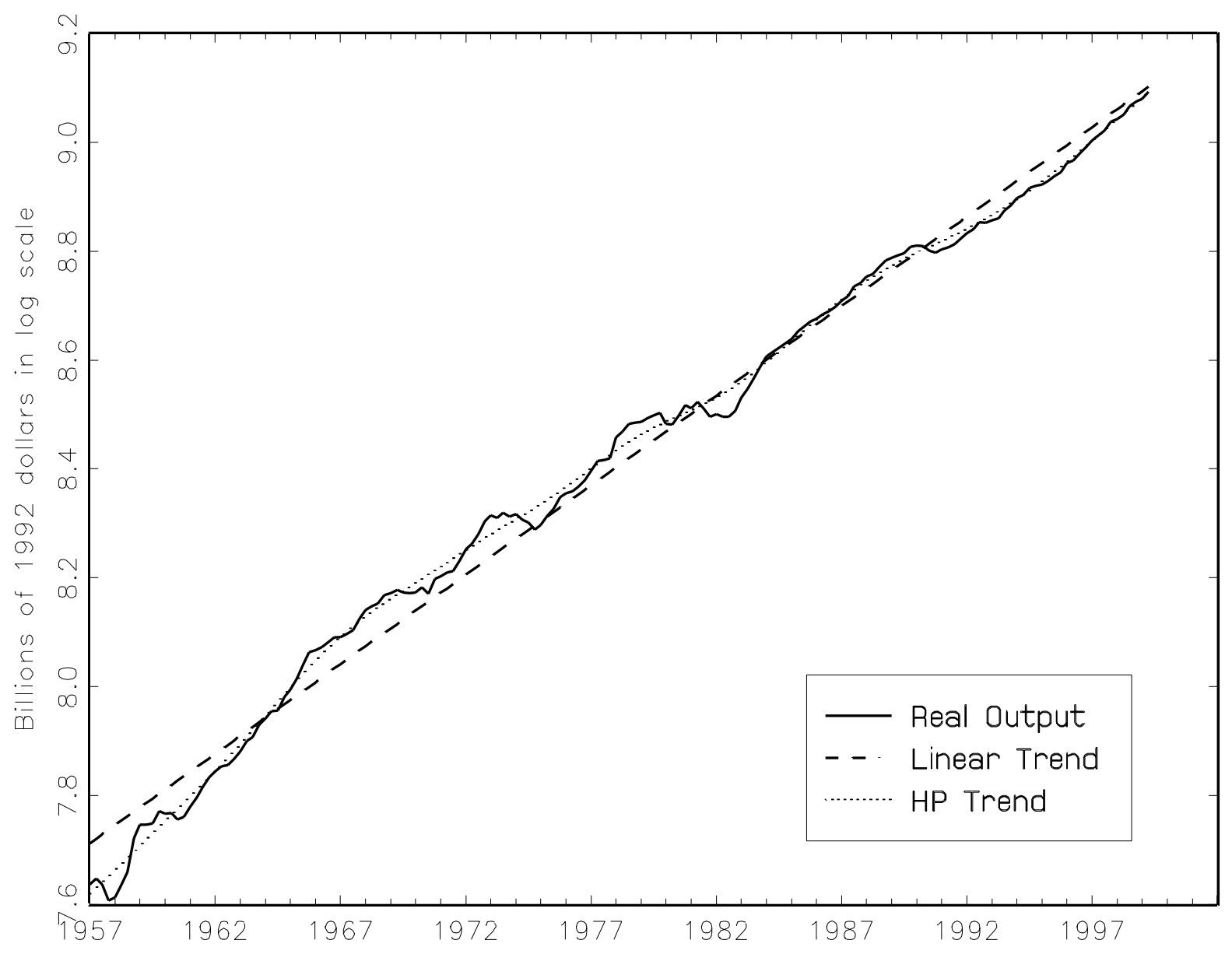


Figure 2

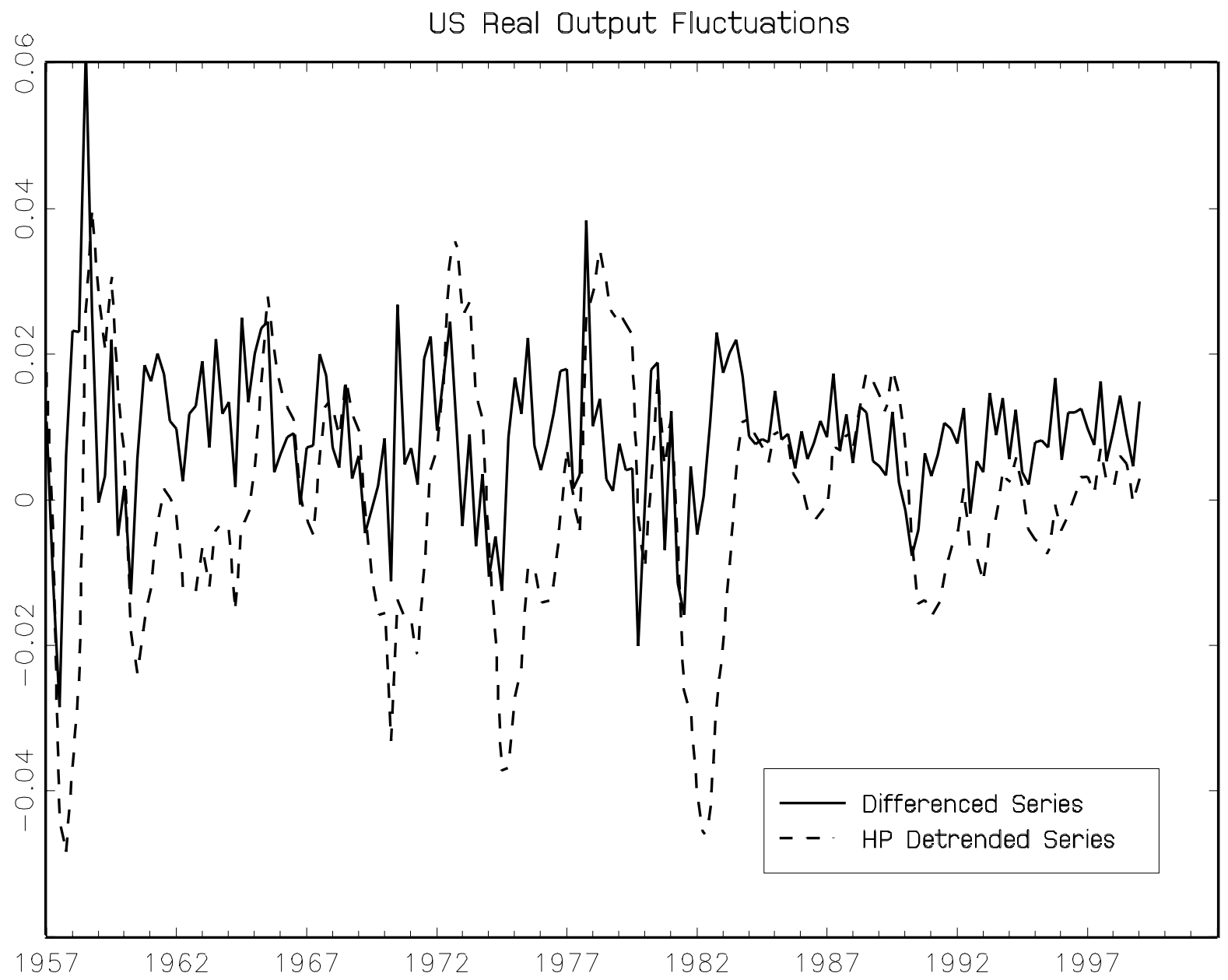

